Article

\title{
Maximum Profit Configurations of Commercial Engines
}

\section{Yiran Chen}

Institute for Advanced Study, Wuhan University, Wuhan 430072, China;

E-Mail: yiranchen90@hotmail.com; Tel.: +86-27-83443326; Fax: +86-27-83638709

Received: 19 April 2011; in revised form: 20 May 2011 / Accepted: 31 May 2011 /

Published: 7 June 2011

\begin{abstract}
An investigation of commercial engines with finite capacity low- and high-price economic subsystems and a generalized commodity transfer law $\left[n \propto \Delta\left(P^{m}\right)\right]$ in commodity flow processes, in which effects of the price elasticities of supply and demand are introduced, is presented in this paper. Optimal cycle configurations of commercial engines for maximum profit are obtained by applying optimal control theory. In some special cases, the eventual state - market equilibrium - is solely determined by the initial conditions and the inherent characteristics of two subsystems; while the different ways of transfer affect the model in respects of the specific forms of the paths of prices and the instantaneous commodity flow, i.e., the optimal configuration.
\end{abstract}

Keywords: finite time thermodynamics; commercial engine; optimal configuration

PACS Codes: 05.70.Ln, 44.40.+a89

\section{Introduction}

In the realm of finite time thermodynamics, two issues are of essential importance-one is to determine the extremum of objective function and study the interrelation of different objective functions, the other is to determine the optimal thermodynamic process for given optimization objectives [1-16]. Curzon and Ahlborn [17] demonstrated that the efficiency at maximum power point is $\eta_{C A}=1-\sqrt{T_{L} / T_{H}}$ for an endoreversible Carnot heat engine operating between two constant temperature reservoirs with Newtonian heat transfer law $[q \propto \Delta(T)]$. Procaccia and Ross [18] proved that in all acceptable cycles, an endoreversible Carnot cycle with larger compression ratio can produce maximum power, i.e., the Curzon-Ahlborn cycle [17] is the optimal configuration with only First and Second Law constraints. Ondrechen et al. [19] studied the optimal cycle configuration of an 
endoreversible heat engine with a finite thermal capacity reservoir and Newtonian heat transfer law for maximum work output. Chen et al. [20] investigated effects of heat leakage on the optimal cycle configuration of a heat engine with a finite thermal capacity reservoir and Newtonian heat transfer law for maximum work output. Linetskii and Tsirlin [21], and Andresen and Gordon [22] considered the minimum entropy generation of heat transfer process with Newtonian heat transfer law in heat exchanger. Based on reference [22], Badescu [23] optimized the heat transfer process with Newtonian heat transfer law for minimum lost available work by choosing the hot bath side as referee environment. Xia et al. [24] optimized the heat transfer process with Newtonian heat transfer law in heat exchanger for entransy dissipation minimization. Nevertheless, generally, heat transfer does not necessarily obey Newtonian heat transfer law, and it may follow other laws. Heat transfer laws not only influence the performance of given thermodynamic processes [25-29], but also influence the optimal configurations of thermodynamic processes for given optimization objectives. Yan et al. [30] investigated the optimal cycle configuration of an endoreversible heat engine with a finite thermal capacity reservoir and the linear phenomenological heat transfer law [ $q \propto \Delta\left(T^{-1}\right)$ ] for maximum work output. Chen et al. [31] investigated effects of heat leakage on the optimal cycle configuration of a heat engine with a finite thermal capacity reservoir and the linear phenomenological heat transfer law for maximum work output. Some studies on the optimal configuration of variable- temperature heat reservoir heat engine for maximum power output were also performed, with the generalized radiative heat transfer law $\left[q \propto \Delta\left(T^{n}\right)\right]$ [32], generalized convective heat transfer law $\left[q \propto(\Delta T)^{m}\right]$ [33], mixed heat resistance [34], and generalized heat transfer law $\left[q \propto\left(\Delta\left(T^{n}\right)\right)^{m}\right]$ [35], respectively. Andresen and Gordon [36] and Badescu [37] further optimized a class of heat transfer processes, with generalized radiative heat transfer law for minimum entropy generation [36] and minimum lost available work [37], respectively. Based on the generalized heat transfer law [ $q \propto\left(\Delta\left(T^{n}\right)\right)^{m}$ ], Chen et al. [38] and Xia et al. [39] derived the optimal temperature configurations of heat transfer processes for minimum entropy generation [38] and minimum lost available work [39]. Xia et al. [40] further investigated the minimum entransy dissipation of heat transfer processes with the generalized radiative heat transfer law.

In the realm of thermodynamics, a thermodynamic system can be described by extensive variables (such as mass, volume, internal energy, and entropy) and intensive variables (such as temperature, and pressure); and heat flux is generated by temperature difference. Similarly, in the realm of economics, variables can also be classified into extensive ones (such as labor, capital, and good) and intensive ones (such as price); moreover, commodity flow is generated by price difference. The striking resemblance of thermodynamics and economics has drawn much attention [2,4,6,7,10,41-49]. Rozonoer [41-43] studied the analogies between reversible thermodynamics and economics in detail, and proposed the term "resource economics" for the analysis of economic system using a thermodynamic approach. Based on the analogies between economics and thermodynamics, Saslow [45] developed economic analogies to the free energy, Maxwell relations, and the Gibbs-Duhem relationship. Salamon et al. [46], Berry et al. [4], Tsirlin [7,10,14], and Mironova et al. [6] addressed the research lines and methods of finite-time thermodynamics into economic analyses. They considered the finite rate commodity flow, and investigated the minimal expenses of resource exchange processes with linear commodity transfer law $[n \propto \Delta(P)]$ and maximal profit rates of constant flow and reciprocal commercial engines (which are analogous to constant flow and reciprocal 
heat engines operating between infinite heat reservoirs in thermodynamics). De Vos [48-50] investigated the analogies among endoreversible heat engines, chemical engines and commercial engines. Based on a generalized commodity transfer law $\left[n \propto \Delta\left(P^{m}\right)\right]$, where the exponent $m$ is closely related to the price elasticity of supply and demand, De Vos $[49,50]$ further investigated the optimal performances of endoreversible commercial engines. Martinas [51] investigated the similarities and differences between irreversible thermodynamics and irreversible economics. Tsirlin [52], Tsirlin et al. [53-55], and Amelkin et al. [56] established an analogy between the processes in microeconomics and irreversible thermodynamics, and defined a physical quality in economics that could be used to measure the irreversibility of commodity exchange processes, i.e., capital dissipation, which is analogous to the physical quality of entropy generation in thermodynamics. Amelkin [57] investigated limit performances of a class of resource exchange processes in complex open microeconomic systems including sequential structure and parallel structure. Tsirlin and Kazakov [58] investigated the optimal cycle configuration of a commercial engine with a finite capacity economic subsystem and the linear transfer law for maximum profit.

This paper will further discuss the issue of commercial engine with a more generalized model by relaxing the assumption of linear transfer law. Actually, commodity flow in this model is assumed to follow the generalized transfer law $\left[n \propto \Delta\left(P^{m}\right)\right]$ [48-50], which in economics represents possibility of different preferences. By applying the methods of finite time thermodynamics, this paper will provide the optimal cycle configuration of the commercial engine and give a straightforward and intuitive demonstration of price convergence in the model.

\section{Model Description}

The model of the commercial engine with finite capacity low-price economic subsystem and finite capacity high-price economic subsystem is illustrated in Figure 1. Both commodity flow and money flow are present in the model; the former flows from the low-price side to the high-price side and the latter flows in the opposite direction. In this paper, commodity flow is considered and it is measured in monetary terms.

Taxation, in particular VAT (value added tax) would give similar loss terms for the monetary flows in the opposite direction. It can be seen as the heat leakage in an irreversible heat engine model [28]. In the endoreversible commercial engine model discussed herein, it is neglected just as did for the endoreversible heat engine model [26,27].

The capacity of the low-price economic subsystem is constant $C_{1}$. The commodity price in the subsystem is $P_{1}$, whose initial value is given by $P_{1}(0)=P_{10}$. In addition, the dynamics of $P_{1}$ satisfies the equation:

$$
C_{1} d P_{1} / d t=-d N_{1} / d t
$$

which is an analogy to the dynamics of temperature of a heat reservoir with finite thermal capacity. It is a reasonable analogy because the behavior of $P_{1}$ described by Equation (1) is compatible with the common assumption in economics of diminishing marginal utility. Similarly, for the finite capacity high-price subsystem, the capacity is constant $C_{2}$, commodity price is $P_{2}$ with initial value $P_{2}\left(t_{1}\right)=P_{20}\left(t_{1}\right.$ is the initial time for selling) and dynamics: 


$$
C_{2} d P_{2} / d t=-d N_{2} / d t
$$

Figure 1. Commercial engine model.

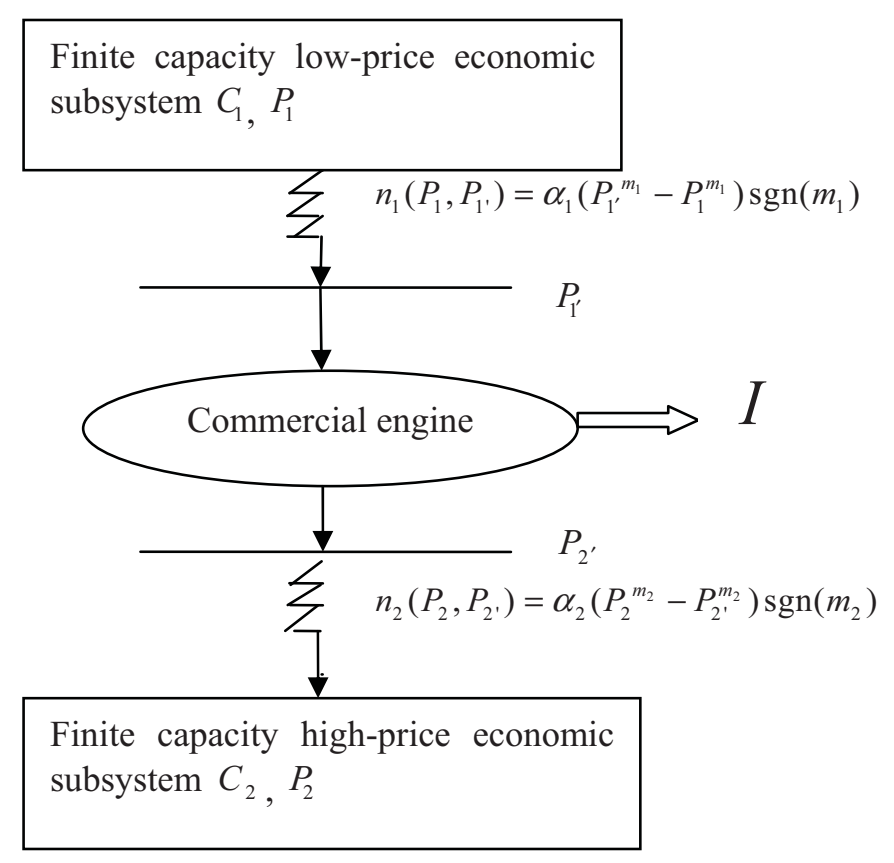

To consider the cases where one side or both sides have infinite capacity, one merely needs to slightly modify the results of model in this paper by taking the limit $C \rightarrow \infty$. Furthermore, the commodity prices of the commercial engine corresponding to low-price and high-price sides are $P_{1}$. and $P_{2}$, respectively, with $P_{1}<P_{1},<P_{2},<P_{2}$.

Different from the linear transfer law $n \propto \Delta(P)$ adopted in [58], commodity flows in the present model are generalized to follow the generalized transfer law $n \propto \Delta\left(P^{m}\right)$ [48-50]:

$$
n_{1}\left(P_{1}, P_{1},\right)=\alpha_{1}\left(P_{1}{ }^{m_{1}}-P_{1}^{m_{1}}\right) \operatorname{sgn}\left(m_{1}\right), \quad n_{2}\left(P_{2}, P_{2}\right)=\alpha_{2}\left(P_{2}^{m_{2}}-P_{2}^{m^{\prime}}\right) \operatorname{sgn}\left(m_{2}\right)
$$

where $n_{1}\left(P_{1}, P_{1},\right)$ and $n_{2}\left(P_{2}, P_{2}\right)$ are commodity flows corresponding to low-price and highprice sides of the commercial engine, $\alpha_{1}(t)$ and $\alpha_{2}(t)$ are the corresponding transfer coefficients, and exponents $m_{1}$ and $m_{2}$ are indicators of price elasticity of supply or demand. To elucidate, elasticity is a measure of the responsiveness of supply or demand to price changes, mathematically:

$$
\varepsilon_{1}=\frac{d n_{1} / n_{1}}{d P_{1^{\prime}} / P_{1^{\prime}}}, \quad \varepsilon_{2}=-\frac{d n_{2} / n_{2}}{d P_{2^{\prime}} / P_{2^{\prime}}}
$$

Substituting Equation (3) into Equation (4) yields:

$$
\mathcal{E}_{1}=\frac{m_{1} P_{1^{\prime}}{ }^{m_{1}}}{P_{1^{\prime}}^{m_{1}}-P_{1}^{m_{1}}}, \quad \mathcal{E}_{2}=\frac{m_{2} P_{2^{\prime}}{ }^{m_{2}}}{P_{2}^{m_{2}}-P_{2^{\prime}}{ }^{m_{2}}}
$$

A one-to-one relationship between the exponent and elasticity is established above. It should be noted that $m_{1}$ and $m_{2}$ don't necessarily have to be the same, because different $m$ 's may represent different preferences of suppliers and demanders.

The amount of commodity exchange in the low-price side and high price side are denoted as $\Delta N_{1}$ 
and $\Delta N_{2}$, respectively. They are given by:

$$
\begin{gathered}
\Delta N_{1}=\int_{0}^{\tau} n_{1}\left(P_{1}, P_{1^{\prime}}\right) d t=\int_{0}^{\tau} \alpha_{1}(t)\left[P_{1^{\prime}}^{m_{1}}(t)-P_{1}^{m_{1}}(t)\right] \operatorname{sgn}\left(m_{1}\right) d t \\
\Delta N_{2}=\int_{0}^{\tau} n_{2}\left(P_{2^{\prime}}, P_{2}\right) d t=\int_{0}^{\tau} \alpha_{2}(t)\left[P_{2}^{m_{2}}-P_{2^{\prime}}^{m_{2}}(t)\right] \operatorname{sgn}\left(m_{2}\right) d t
\end{gathered}
$$

where $\tau$ is the given cycle period. Additionally, market equilibrium condition requires that:

$$
\Delta N_{1}=\Delta N_{2}=\Delta N
$$

It is further assumed that purchase and selling are separate and successive processes. At time $t\left(0<t<t_{1}\right)$, the commercial engine purchases commodity from the low-price subsystem; and at time $t\left(t_{1}<t<\tau\right)$, the commercial engine sells commodity to the high-price subsystem. Therefore, $\alpha_{1}(t)$ and $\alpha_{2}(t)$ have the following forms:

$$
\alpha_{1}(t)=\left\{\begin{array}{ll}
\alpha_{1}, & 0 \leq t \leq t_{1} \\
0, & t_{1} \leq t \leq \tau
\end{array}, \quad \alpha_{2}(t)=\left\{\begin{array}{lc}
0, & 0 \leq t \leq t_{1} \\
\alpha_{2}, & t_{1} \leq t \leq \tau
\end{array}\right.\right.
$$

where $\alpha_{1}$ and $\alpha_{2}$ are positive constants.

Finally, profit gained by the commercial engine is given by:

$$
I=\int_{0}^{\tau}\left[P_{2^{\prime}}(t) n_{2}\left(P_{2^{\prime}}, P_{2}\right)-P_{1^{\prime}}(t) n_{1}\left(P_{1}, P_{1^{\prime}}\right)\right] d t
$$

\section{Optimization}

The optimization problem for the commercial engine is to maximize its profit with the constraints of market equilibrium conditions and the predetermined dynamics of prices in the two economic subsystems. Mathematically, the problem amounts to determine the optimal paths of $P_{1^{\prime}}$ and $P_{2^{\prime}}$, the optimal values of $t_{1}$ and $\Delta N$ to maximize Equation (10) subject to Equations (1), (2) and (8).

Following the method adopted in [58], optimization problem is decomposed into two sub-problems.

\subsection{Problem 1}

$$
\begin{gathered}
\max I^{-}=\int_{0}^{t_{1}}-\alpha_{1}\left(P_{1^{\prime}}^{m_{1}}-P_{1}^{m_{1}}\right) \operatorname{sgn}\left(m_{1}\right) P_{1}, d t \\
\text { s.t } d P_{1} / d t=\alpha_{1}\left(P_{1}{ }^{m_{1}}-P_{1}{ }^{m_{1}}\right) \operatorname{sgn}\left(m_{1}\right) / C_{1} \\
\int_{0}^{t_{1}} \alpha_{1}\left(P_{1^{\prime}}^{m_{1}}-P_{1}^{m_{1}}\right) \operatorname{sgn}\left(m_{1}\right) d t=\Delta N_{1}=\Delta N
\end{gathered}
$$

Equation (12) is obtained by substituting Equation (6) into Equation (1). Substituting Equation (12) into Equations (11) and (13) yields:

$$
\begin{gathered}
\max \quad I^{-}=\int_{P_{10}}^{P_{1}\left(t_{1}\right)}-C_{1} P_{1}, d P_{1} \\
\int_{P_{10}}^{P_{1}\left(t_{1}\right)} C_{1} d P_{1}=\Delta N
\end{gathered}
$$


Equation (12) itself can be transformed to:

$$
\int_{P_{10}}^{P_{1}\left(t_{1}\right)} \frac{C_{1}}{\alpha_{1}\left(P_{1^{\prime}}^{m_{1}}-P_{1}^{m_{1}}\right) \operatorname{sgn}\left(m_{1}\right)} d P_{1}=t_{1}
$$

The problem now becomes maximizing Equation (14) subject to Equations (15) and (16). The corresponding modified Lagrangian function is given by:

$$
L_{1}=C_{1}\left[-P_{1^{\prime}}+\lambda_{1}+\frac{\lambda_{2}}{\alpha_{1}\left(P_{1^{\prime}}^{m_{1}}-P_{1}^{m_{1}}\right) \operatorname{sgn}\left(m_{1}\right)}\right]
$$

where $\lambda_{1}$ and $\lambda_{1}$ are Lagrangian multipliers.

First order condition with respect to $P_{1^{\prime}}$ yields

$$
P_{1}^{m_{1}}-P_{1}^{m_{1}}=k_{1} P_{1}^{\left(m_{1}-1\right) / 2}
$$

where $k_{1}$ is a constant to be determined.

Combining Equation (18) with Equation (12) yields:

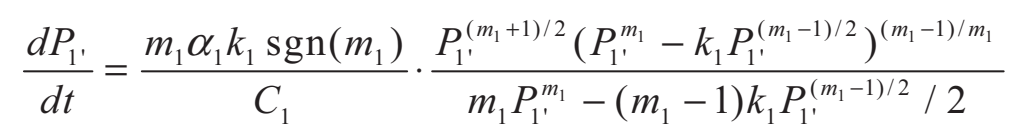

The dynamics of $P_{1^{\prime}}$ are uniquely characterized by Equation (19), which, combined with Equations (18), (13) and the initial value of $P_{1}$, determines the paths of both $P_{1^{\prime}}$ and $P_{1}$.

\subsection{Problem 2}

$$
\begin{gathered}
\max I^{+}=\int_{t_{1}}^{\tau} \alpha_{2}\left(P_{2}^{m_{2}}-P_{2^{\prime}}^{m_{2}}\right) \operatorname{sgn}\left(m_{2}\right) P_{2}, d t \\
\text { s.t. } d P_{2} / d t=-\alpha_{2}\left(P_{2}^{m_{1}}-P_{2^{\prime}}^{m_{1}}\right) \operatorname{sgn}\left(m_{2}\right) / C_{2} \\
\int_{t_{1}}^{\tau} \alpha_{2}\left(P_{2}^{m_{2}}-P_{2^{\prime}}^{m_{2}}\right) \operatorname{sgn}\left(m_{2}\right) d t=\Delta N_{2}=\Delta N
\end{gathered}
$$

Equation (21) is obtained by substituting Equation (7) into Equation (2). Substituting Equation (21) into Equations (20) and (22) yields:

$$
\begin{gathered}
\max \quad I^{+}=-\int_{P_{20}}^{P_{2}(\tau)} C_{2} P_{2}, d P_{2} \\
-\int_{P_{20}}^{P_{2}(\tau)} C_{2} d P_{2}=\Delta N
\end{gathered}
$$

Equation (21) itself can be transformed to:

$$
-\int_{P_{20}}^{P_{2}(\tau)} \frac{C_{2}}{\alpha_{2}\left(P_{2}^{m_{2}}-P_{2^{\prime}}^{m_{2}}\right) \operatorname{sgn}\left(m_{2}\right)} d P_{2}=\tau-t_{1}
$$

The problem now becomes maximizing Equation (23) subject to Equations (24) and (25). The corresponding modified Lagrangian function is given by:

$$
L_{2}=-C_{2}\left[P_{2^{\prime}}+\lambda_{3}+\frac{\lambda_{4}}{\alpha_{2}\left(P_{2}^{m_{2}}-P_{2^{\prime}}^{m_{2}}\right) \operatorname{sgn}\left(m_{2}\right)}\right]
$$


where $\lambda_{3}$ and $\lambda_{4}$ are Lagrangian multipliers. First order condition with respect to $P_{2^{\prime}}$ yields:

$$
P_{2}^{m_{2}}-P_{2^{\prime}}^{m_{2}}=k_{2} P_{2}^{\left(m_{2}-1\right) / 2}
$$

where $k_{2}$ is a constant to be determined.

Combining Equation (27) with Equation (21) yields:

$$
\frac{d P_{2^{\prime}}}{d t}=-\frac{m_{2} \alpha_{2} k_{2} \operatorname{sgn}\left(m_{2}\right)}{C_{2}} \cdot \frac{P_{2^{\prime}}^{\left(m_{1}+1\right) / 2}\left(P_{2^{\prime}}^{m_{2}}+k_{2} P_{2^{\prime}}^{\left(m_{2}-1\right) / 2}\right)^{\left(m_{1}-1\right) / m_{1}}}{m_{2} P_{2^{\prime}}^{m_{2}}+\left(m_{2}-1\right) k_{2} P_{2^{\prime}}^{\left(m_{2}-1\right) / 2} / 2}
$$

The dynamics of $P_{2^{\prime}}$ are uniquely characterized by Equation (28), which, combined with Equations (22), (27) and the initial value of $P_{2}$, determines the paths of both $P_{2^{\prime}}$ and $P_{2}$. In sum, the optimal paths of $P_{1^{\prime}}$ and $P_{2^{\prime}}$ are described by Equations (19) and (28). However, analytical solutions to these differential equations exist only for a few exponents such as 1 and -1 . For other exponents which do not admit analytical solutions, numerical method should be adopted.

To further determine the optimal values of $\Delta N, t_{1}$, and $I$, one merely needs to substitute the paths of $P_{1^{\prime}}, P_{1}, P_{2^{\prime}}$ and $P_{2}$ into Equation (10) and solve the system of first order conditions.

4. Special case with $m_{1}=1$ and $m_{2}=1$

\subsection{Analytical Solutions}

For problem 1, Equations (19), (18) and (13) are simplified to $\frac{d P_{1^{\prime}}}{d t}=\frac{\alpha_{1} k_{1}}{C_{1}}, P_{1^{\prime}}-P_{1}=k_{1}$ and $\int_{0}^{t_{1}} \alpha_{1}\left(P_{1^{\prime}}-P_{1}\right) d t=\Delta N$, respectively, in this case. Solving the system gives the paths of $P_{1}$ and $P_{1^{\prime}}$, respectively:

$$
\begin{gathered}
P_{1}=\left(\Delta N / C_{1} t_{1}\right) t+P_{10} \quad\left(0 \leq t \leq t_{1}\right) \\
P_{1^{\prime}}=\left(\Delta N / C_{1} t_{1}\right) t+P_{10}+\Delta N / \alpha_{1} t_{1} \quad\left(0 \leq t \leq t_{1}\right)
\end{gathered}
$$

For problem 2, Equations (28), (27) and (22) are simplified to $\frac{d P_{2}}{d t}=\frac{\alpha_{2} k_{2}}{C_{2}}, P_{2},-P_{2}=k_{2}$ and $\int_{t_{1}}^{\tau} \alpha_{2}\left(P_{2}-P_{2^{\prime}}\right) d t=\Delta N$, respectively, in this case. Solving the system gives the paths of $P_{2}$ and $P_{2^{\prime}}$, respectively:

$$
\begin{gathered}
P_{2}=-\left[\Delta N / C_{2}\left(\tau-t_{1}\right)\right]\left(t-t_{1}\right)+P_{20} \quad\left(t_{1} \leq t \leq \tau\right) \\
P_{2^{\prime}}=-\left[\Delta N / C_{2}\left(\tau-t_{1}\right)\right]\left(t-t_{1}\right)+P_{20}-\Delta N / \alpha_{2}\left(\tau-t_{1}\right) \quad\left(t_{1} \leq t \leq \tau\right)
\end{gathered}
$$

Substituting Equations (29), (30), (31) and (32) into Equation (10) yields:

$$
I=\left(P_{20}-P_{10}\right) \Delta N-\left[\left(1 / 2 C_{2}+1 / 2 C_{1}+1 / \alpha_{2}\left(\tau-t_{1}\right)+1 / \alpha_{1} t_{1}\right] \Delta N\right.
$$

First order condition $\delta I / \delta t_{1}=0$ yields:

$$
t_{1}^{*}=\frac{\sqrt{\alpha_{2}} \tau}{\sqrt{\alpha_{1}}+\sqrt{\alpha_{2}}}
$$


Substituting Equation (29) into the First order condition $\delta I / \delta(\Delta N)=0$ yields:

$$
\Delta N^{*}=\frac{\left(P_{20}-P_{10}\right) \tau}{\tau\left(1 / C_{1}+1 / C_{2}\right)+2\left(\sqrt{\alpha_{1}}+\sqrt{\alpha_{2}}\right)^{2} /\left(\alpha_{1} \alpha_{2}\right)}
$$

Therefore:

$$
I_{\max }=\frac{\left(P_{20}-P_{10}\right)^{2} \tau}{2 \tau\left(1 / C_{1}+1 / C_{2}\right)+4\left(\sqrt{\alpha_{1}}+\sqrt{\alpha_{2}}\right)^{2} /\left(\alpha_{1} \alpha_{2}\right)}
$$

Substituting Equations (34) and (35) into Equations (29), (30), (31) and (32) yields, respectively:

$$
\begin{gathered}
P_{1}^{*}=\frac{\left(P_{20}-P_{10}\right)\left(\sqrt{\alpha_{1}}+\sqrt{\alpha_{2}}\right)}{C_{1} \sqrt{\alpha_{2}}\left[\tau\left(1 / C_{1}+1 / C_{2}\right)+2\left(\sqrt{\alpha_{1}}+\sqrt{\alpha_{2}}\right)^{2} /\left(\alpha_{1} \alpha_{2}\right)\right]} t+P_{10} \quad\left(0 \leq t \leq t_{1}\right) \\
P_{1^{*}}{ }^{*}=\frac{\left(P_{20}-P_{10}\right)\left(\sqrt{\alpha_{1}}+\sqrt{\alpha_{2}}\right)}{C_{1} \sqrt{\alpha_{2}}\left[\tau\left(1 / C_{1}+1 / C_{2}\right)+2\left(\sqrt{\alpha_{1}}+\sqrt{\alpha_{2}}\right)^{2} /\left(\alpha_{1} \alpha_{2}\right)\right]}\left(t+\frac{C_{1}}{\alpha_{1}}\right)+P_{10} \quad\left(0 \leq t \leq t_{1}\right) \\
P_{2}^{*}=\frac{-\left(P_{20}-P_{10}\right)\left(\sqrt{\alpha_{1}}+\sqrt{\alpha_{2}}\right)}{C_{2} \sqrt{\alpha_{1}}\left[\tau\left(1 / C_{1}+1 / C_{2}\right)+2\left(\sqrt{\alpha_{1}}+\sqrt{\alpha_{2}}\right)^{2} /\left(\alpha_{1} \alpha_{2}\right)\right]}\left(t-\frac{\sqrt{\alpha_{2}} \tau}{\sqrt{\alpha_{1}}+\sqrt{\alpha_{2}}}\right)+P_{20} \quad\left(t_{1} \leq t \leq \tau\right) \\
P_{2^{*}}{ }^{*}=\frac{-\left(P_{20}-P_{10}\right)\left(\sqrt{\alpha_{1}}+\sqrt{\alpha_{2}}\right)}{C_{2} \sqrt{\alpha_{1}}\left[\tau\left(1 / C_{1}+1 / C_{2}\right)+2\left(\sqrt{\alpha_{1}}+\sqrt{\alpha_{2}}\right)^{2} /\left(\alpha_{1} \alpha_{2}\right)\right]}\left(t-\frac{\sqrt{\alpha_{2}} \tau}{\sqrt{\alpha_{1}}+\sqrt{\alpha_{2}}}+\frac{C_{2}}{\alpha_{2}}\right)+P_{20} \quad\left(t_{1} \leq t \leq \tau\right)
\end{gathered}
$$

\subsection{Results and Discussion}

It is revealed above that both $\mathrm{P}_{1}{ }^{*}$ and $\mathrm{P}_{1^{\prime}}{ }^{*}$ increase linearly in the time, while both $\mathrm{P}_{2}{ }^{*}$ and $\mathrm{P}_{2^{\prime}}{ }^{*}$ decrease linearly in the time; commodity flows $n_{1}\left(P_{1}, P_{1}{ }^{\prime}\right)$ and $n_{2}\left(P_{2}, P_{2}\right)$ are constants over time; the optimal exchange time $t_{1}{ }^{*}$ is determined only by ratio of the transfer coefficients $\alpha_{1}$ and $\alpha_{2}$.

The most enlightening implication of the result is the convergence of the eventual values of $\mathrm{P}_{1}^{*}$, $\mathrm{P}_{1^{\prime}}{ }^{*}, \mathrm{P}_{2}{ }^{*}$ and $\mathrm{P}_{2^{\prime}}{ }^{*}$. Mathematically:

$$
\lim _{\tau \rightarrow \infty} \mathrm{P}_{1}^{*}\left(\mathrm{t}_{1}\right)=\lim _{\tau \rightarrow \infty} \mathrm{P}_{1^{\prime}}{ }^{*}\left(\mathrm{t}_{1}\right)=\lim _{\tau \rightarrow \infty} \mathrm{P}_{1^{\prime}}{ }^{*}\left(\mathrm{t}_{1}\right)=\lim _{\tau \rightarrow \infty} \mathrm{P}_{2^{\prime}}{ }^{*}(\tau)=\frac{C_{1} \mathrm{P}_{10}+C_{2} P_{20}}{C_{1}+C_{2}}
$$

The common limit is exactly equilibrium price which completely clears the market, i.e.:

$$
P_{e}=\frac{C_{1} \mathrm{P}_{10}+C_{2} P_{20}}{C_{1}+C_{2}}
$$

which is a weighted average of the initial prices of two subsystems, and the weights are the corresponding capacities. Larger capacity indicates larger market power, therefore equilibrium price is more biased to the initial price of the party with larger capacity. Especially, if one side has infinite capacity, the equilibrium price will be the same as its initial price.

Additionally, The convergence of $P_{1}$ * and $P_{2}$ * also indicates that the instantaneous profit gained by the commercial engine diminishes to 0 as the cycle period approaches infinity, which further indicates that the total profit earned cannot be infinite. Equation (36) serves as an apt substantiation of this point: 


$$
\lim _{\tau \rightarrow \infty} I_{\max }=\frac{\left(P_{20}-P_{10}\right)^{2}}{2\left(1 / C_{1}+1 / C_{2}\right)}
$$

Finally, price convergence denies long-existing price discrepancy in a pure exchange market without exogenous interventions. Another simple but profound implication is that the profit-maximizing behavior of a commercial engine induces an optimal outcome for the market. In other words, the commercial engine, motivated by its own interest, acts as catalyst in the process of reducing price discrepancy and reaching market equilibrium. However, its existence cannot be permanent since its profit diminishes to 0 with time. In this perspective, the commercial engine can be viewed as an arbitrager whose profit-seeking action results in price parity; and the whole model simulates the dynamic process of the determination of equilibrium price.

5. Special Case with $m_{1}=-1$ and $m_{2}=-1$

\subsection{Analytical Solutions}

For problem 1, Equations (19), (18) and (13) are simplified to $\frac{d P_{1^{\prime}}}{d t}=\frac{\alpha_{1} k_{1}\left(k_{1}-1\right)}{C_{1}} P_{1^{\prime}}{ }^{-1}$, $P_{1^{\prime}}^{-1}-P_{1}^{-1}=k_{1} P_{1^{\prime}}^{-1}$ and $-\int_{0}^{t_{1}} \alpha_{1}\left(P_{1^{\prime}}^{-1}-P_{1}^{-1}\right) d t=\Delta N$, respectively. Solving the system gives the paths of $P_{1}$ and $P_{1^{\prime}}$, respectively:

$$
\begin{gathered}
P_{1}=\frac{\sqrt{\Delta N t_{1}\left(\Delta N+2 C P_{10}\right) t+C^{2} t_{1}^{2} P_{10}^{2}}}{C t_{1}} \\
P_{1^{\prime}}=\frac{2 \alpha_{1} \sqrt{\Delta N t_{1}\left(\Delta N+2 C P_{10}\right) t+C^{2} t_{1}^{2} P_{10}^{2}}}{2 C \alpha_{1} t_{1}-\left(\Delta N^{2}+2 \Delta N C P_{10}\right)}
\end{gathered}
$$

For problem 2, Equations (28), (27) and (22) are simplified to $\frac{d P_{2^{\prime}}}{d t}=\frac{\alpha_{1} k_{2}\left(k_{2}+1\right)}{C_{2}} P_{2^{\prime}}{ }^{-1}$, $P_{2}^{-1}-P_{2^{\prime}}^{-1}=k_{2} P_{2}^{-1}$ and $-\int_{t_{1}}^{\tau} \alpha_{1}\left(P_{2}^{-1}-P_{2^{\prime}}^{-1}\right) d t=\Delta N$, respectively. Solving the system gives the paths of $P_{2}$ and $P_{2^{\prime}}$, respectively:

$$
\begin{gathered}
P_{2}=\frac{\sqrt{\left(\Delta N^{2}-2 \Delta N C_{2} P_{20}\right)\left(\tau-t_{1}\right)\left(t-t_{1}\right)+C_{2}^{2}\left(\tau-t_{1}\right)^{2} P_{20}^{2}}}{C_{2}\left(\tau-t_{1}\right)} \\
P_{2^{\prime}}=\frac{2 \alpha_{2} \sqrt{\left(\Delta N^{2}-2 \Delta N C_{2} P_{20}\right)\left(\tau-t_{1}\right)\left(t-t_{1}\right)+C_{2}^{2}\left(\tau-t_{1}\right)^{2} P_{20}^{2}}}{-\Delta N^{2}+2 \Delta N C_{2} P_{20}+2 C_{2} \alpha_{2}\left(\tau-t_{1}\right)}
\end{gathered}
$$

Substituting Equations (44), (45), (46) and (47) into Equation (10) yields:

$$
I=\frac{\alpha_{2}\left(\tau-t_{1}\right)\left(\Delta N^{2}-2 \Delta N C_{2} P_{20}\right)}{\Delta N^{2}-2 \Delta N C_{2} P_{20}-2 C_{2} \alpha_{2}\left(\tau-t_{1}\right)}+\frac{\alpha_{1} t_{1}\left(\Delta N^{2}+2 \Delta N C_{1} P_{10}\right)}{\Delta N^{2}+2 \Delta N C_{1} P_{10}-2 C_{1} \alpha_{1} t_{1}}
$$

The optimal $t_{1}{ }^{*}$ and $\Delta N^{*}$ are jointly determined by first order conditions $\partial I / \partial t_{1}=0$ and $\partial I / \partial(\Delta N)=0$. However, the system of polynomials cannot be solved explicitly. 


\subsection{Results and Discussion}

This section focuses on discussing the behaviors of $\mathrm{P}_{1}{ }^{*}, \mathrm{P}_{1^{\prime}}{ }^{*}, \mathrm{P}_{2}{ }^{*}$ and $\mathrm{P}_{2^{\prime}}{ }^{*}$ as $\tau \rightarrow \infty$. From Equations (44) and (46) one can obtain:

$$
\begin{gathered}
P_{1}^{*}\left(t_{1}\right)=\frac{\Delta N^{*}+C_{1} P_{10}}{C_{1}}=\frac{\Delta N^{*}}{C_{1}}+P_{10} \\
P_{2}^{*}(\tau)=\frac{-\Delta N^{*}+C_{2} P_{20}}{C_{2}}=\frac{-\Delta N^{*}}{C_{2}}+P_{20}
\end{gathered}
$$

They depend on $\Delta N^{*}$ which cannot be solved explicitly.

To proceed, first suppose there does exist an optimal solution where $\mathrm{P}_{1}{ }^{*}, \mathrm{P}_{1^{\prime}}{ }^{*}, \mathrm{P}_{2}{ }^{*}$ and $\mathrm{P}_{2^{\prime}}{ }^{*}$ converge eventually. Then $\Delta N^{*}$ is bounded; and thus as $\tau \rightarrow \infty$, Equation (48) becomes:

$$
\lim _{\tau \rightarrow \infty} I=\frac{\Delta N^{2}-2 \Delta N C_{2} P_{20}}{-2 C_{2}}+\frac{\Delta N^{2}+2 \Delta N C_{1} P_{10}}{-2 C_{1}}
$$

First order condition $\partial \lim _{\tau \rightarrow \infty} I / \partial(\Delta N)=0$ yields:

$$
\lim _{\tau \rightarrow \infty} \Delta N^{*}=\frac{P_{20}-P_{10}}{1 / C_{1}+1 / C_{2}}
$$

To check whether the $\Delta N^{*}$ determined by Equation (52) supports such an optimal solution, substitute Equation (52) into Equations (49) and (50):

$$
\lim _{\tau \rightarrow \infty} \mathrm{P}_{1}^{*}\left(\mathrm{t}_{1}\right)=\lim _{\tau \rightarrow \infty} \mathrm{P}_{2}^{*}(\tau)=\frac{C_{1} \mathrm{P}_{10}+C_{2} P_{20}}{C_{1}+C_{2}}
$$

Since $P_{1}<P_{1},<P_{2},<P_{2}$, there must be:

$$
P_{e}=\lim _{\tau \rightarrow \infty} \mathrm{P}_{1}^{*}\left(\mathrm{t}_{1}\right)=\lim _{\tau \rightarrow \infty} \mathrm{P}_{1^{\prime}}{ }^{*}\left(\mathrm{t}_{1}\right)=\lim _{\tau \rightarrow \infty} \mathrm{P}_{1^{\prime}}{ }^{*}\left(\mathrm{t}_{1}\right)=\lim _{\tau \rightarrow \infty} \mathrm{P}_{2^{\prime}}{ }^{*}(\tau)=\frac{C_{1} \mathrm{P}_{10}+C_{2} P_{20}}{C_{1}+C_{2}}
$$

It is revealed that all four prices share common limit; therefore convergence of prices is actually an optimal solution to this problem. The corresponding maximum profit is given by:

$$
\lim _{\tau \rightarrow \infty} I_{\max }=\frac{\left(P_{20}-P_{10}\right)^{2}}{2\left(1 / C_{1}+1 / C_{2}\right)}
$$

Comparing the results of this case with $m_{1}=m_{2}=-1$ with those of the previous case with $m_{1}=m_{2}=1$, one finds that the equilibrium price $P_{e}$, the optimal amount of commodity exchange $\Delta N^{*}$, and the maximum profit $I_{\mathrm{max}}$ are the same. It should be noted that this phenomenon is not a coincidence. Actually, in this model, the eventual state - market equilibrium - is solely determined by the initial conditions and the inherent characteristics of two subsystems; while the different ways of transfer (reflected by different values of $m_{1}$ and $m_{2}$ ) affect the model in respects of the specific forms of the paths of prices and the instantaneous commodity flow, i.e., the optimal configuration. 


\section{Conclusions}

Commercial engines with finite capacity low-price economic subsystems and a generalized commodity transfer law $\left[n \propto \Delta\left(P^{m}\right)\right]$ during commodity flow processes, in which the effects of the price elasticities of supply and demand are introduced, are investigated in this paper. The optimal cycle configurations of the commercial engines for maximum profit are obtained by applying optimal control theory. The optimal cycle configuration of the commercial engine with the linear transfer law $[n \propto \Delta P$ ] is that both the price estimation of finite capacity low-price economic subsystem and the commodity-buying price of the commercial engine change with time linearly and the difference between them is a constant, and the selling price of the commercial engine is a constant when it exchanges commodity with the infinite capacity high-price economic subsystem. The optimal cycle configuration of the commercial engine with the transfer law $\left[n \propto \Delta\left(P^{-1}\right)\right]$ is that both the price estimation of finite capacity low-price economic subsystem and the commodity-buying price of the commercial engine change with time non-linearly and the ratio between them is a constant, and the selling price of the commercial engine is a constant when it exchanges commodity with the infinite capacity high-price economic subsystem. The research in this paper further extends the research lines and methods of finite time thermodynamics to applications in fields of non-conventional thermodynamics. It is worthwhile to note that several authors [60-64] have criticized finite time thermodynamics (emphasis on the endoreversible model and the corresponding study results) in recent years. The responses to those articles can be seen in [65-69], especially, Chen et al. [67].

\section{Acknowledgements}

The author wishes to thank the reviewers for their careful, unbiased and constructive suggestions, which led to this revised manuscript.

\section{References}

1. Andresen, B.; Berry, R.S.; Ondrechen, M.J.; Salamon, P. Thermodynamics for processes in finite time. Acc. Chem. Res. 1984, 17, 266-271.

2. Sieniutycz, S.; Salamon, P. Advances in Thermodynamics. Volume 4: Finite Time Thermodynamics and Thermoeconomics; Taylor \& Francis: New York, NY, USA, 1990.

3. Bejan, A. Entropy generation minimization: The new thermodynamics of finite-size device and finite-time processes. J. Appl. Phys. 1996, 79, 1191-1218.

4. Berry, R.S.; Kazakov, V.A.; Sieniutycz, S.; Szwast, Z.; Tsirlin, A.M. Thermodynamic Optimization of Finite Time Processes; Wiley: Chichester, UK, 1999.

5. Chen, L.; Wu, C.; Sun, F. Finite time thermodynamic optimization or entropy generation minimization of energy systems. J. Non-Equil. Thermodyn. 1999, 24, 327-359.

6. Mironova, V.A.; Amelkin, S.A.; Tsirlin, A.M. Mathematical Methods of Finite Time Thermodynamics; Khimia: Moscow, Russia, 2000.

7. Tsirlin, A.M. Optimization Methods in Thermodynamics and Microeconomics; Nauka: Moscow, Russia, 2002. 
8. Hoffman, K.H.; Burzler, J.; Fischer, A.; Schaller, M.; Schubert, S. Optimal process pathes for endoreversible systems. J. Non-Equil. Thermodyn. 2003, 28, 233-268.

9. Sieniutycz, S. Thermodynamic limits on production or consumption of mechanical energy in practical and industry systems. Progr. Energ. Combust. Sci. 2003, 29, 193-246.

10. Tsirlin, A.M. Irreversible Estimates of Limiting Possibilities of Thermodynamic and Microeconomic Systems; Nauka: Moscow, Russia, 2003.

11. Chen, L.; Sun, F. Advances in Finite Time Thermodynamics: Analysis and optimization; Nova Science Publishers: New York, NY, USA, 2004.

12. Durmayaz, A.; Sogut, O.S.; Sahin, B.; Yavuz, H. Optimization of thermal systems based on finitetime thermodynamics and thermoeconomics. Progr.s Energ. Combust. Sci. 2004, 30, 175-217.

13. Chen, L. Finite Time Thermodynamic Analysis of Irreversible Progresses and Cycles; High Education Press: Beijing, China, 2005.

14. Tsirlin, A.M. Optimal processes in open controllable macrosystems. Autom. Rem. Contr. 2006, $67,132-147$.

15. de Vos, A. Thermodynamics of Solar Energy Conversion; Wiley-VCH Verlag: Berlin, Germany, 2008.

16. Sieniutycz, S.; Jezowski, J. Energy Optimization in Process Systems; Elsevier: Oxford, UK, 2009.

17. Curzon, F.L.; Ahlborn, B. Efficiency of a Carnot engine at maximum power output. Am. J. Phys. 1975, 43, 22-24.

18. Cutowicz-Krusin, D.; Procaccia, J.; Ross, J. On the efficiency of rate process: Power and efficiency of heat engines. J. Chem. Phys. 1978, 69, 3898-3906.

19. Ondrechen, M.J.; Rubin, M.H.; Band, Y.B. The generalized Carnot cycles: A working fluid operating in finite time between heat sources and sinks. J. Chem. Phys. 1983, 78, 4721-4727.

20. Chen, L.; Zhou, S.; Sun, F.; Wu, C. Optimal configuration and performance of heat engines with heat leak and finite heat capacity. Open Syst. Inform. Dynam. 2002, 9, 85-96.

21. Linetskii, S.B.; Tsirlin, A.M. Evaluating thermodynamic efficiency and optimizing heat exchangers. Therm. Eng. 1988, 35, 593-597.

22. Andresen, B.; Gordon, J.M. Optimal heating and cooling strategies for heat exchanger design. J. Appl. Phys. 1992, 71, 76-80.

23. Badescu, V. Optimal strategies for steady state heat exchanger operation. J. Phys. D: Appl. Phys. 2004, 37, 2298-2304.

24. Xia, S.; Chen, L.; Sun, F. Optimization for entransy dissipation minimization in heat exchanger. Chin. Sci. Bull. 2009, 54, 3587-3595.

25. de Vos, A. Efficiency of some heat engines at maximum power conditions. Am. J. Phys. 1985, 53, 570-573.

26. Chen, L.; Yan, Z. The effect of heat transfer law on the performance of a two-heat-source endoreversible cycle. J. Chem. Phys. 1989, 90, 3740-3743.

27. Chen, L.; Sun, F.; Wu, C. The influence of heat transfer law on the endoreversible Carnot refrigerator. J. Inst. Energ. 1996, 69, 96-100.

28. Chen, L.; Sun, F.; Wu, C. Effect of heat transfer law on the performance of a generalized irreversible Carnot engine. J. Phys. D: Appl. Phys. 1999, 32, 99-105. 
29. Huleihil, M.; Andresen, B. Convective heat transfer law for an endoreversible engine. J. Appl. Phys. 2006, 100, 014911.

30. Yan, Z.; Chen, J. Optimal performance of a generalized Carnot cycle for another linear heat transfer law. J. Chem. Phys. 1990, 92, 1994-1998.

31. Chen, L.; Sun, F.; Wu, C. Optimal configuration of a two-heat-reservoir heat- engine with heat leak and finite thermal capacity. Appl. Energ. 2006, 83, 71-81.

32. Xiong, G.; Chen, J.; Yan, Z. The effect of heat transfer law on the performance of a generalized Carnot cycle. J. Xiamen Univ. (Nature Science) 1989, 28, 489-494.

33. Chen, L.; Zhu, X.; Sun, F.; Wu, C. Optimal configurations and performance for a generalized Carnot cycle assuming the heat transfer law $Q \propto(\Delta T)^{m}$. Appl. Energ. 2004, 78, 305-313.

34. Chen, L.; Zhu, X.; Sun, F.; Wu, C. Effect of mixed heat resistance on the optimal configuration and performance of a heat-engine cycle. Appl. Energ. 2006, 83, 537-544.

35. Li, J.; Chen, L.; Sun, F. Optimal configuration for a finite high-temperature source heat engine cycle with complex heat transfer law. Sci. China Ser. G 2009, 52, 587-592.

36. Andresen, B.; Gordon, J.M. Optimal paths for minimizing entropy generation in a common class of finite time heating and cooling processes. Int. J. Heat Fluid Flow 1992, 13, 294-299.

37. Badescu, V. Optimal paths for minimizing lost available work during usual heat transfer processes. J. Non-Equil. Thermodyn. 2004, 29, 53-73.

38. Chen, L., Xia, S.; Sun, F. Optimal paths for minimizing entropy generation during heat transfer processes with a generalized heat transfer law. J. Appl. Phys. 2009, 105, 044907.

39. Xia, S.; Chen, L.; Sun, F. Optimization for minimizing lost available work during heat transfer processes with complex heat transfer law. Braz. J. Phys. 2009, 39, 98-105.

40. Xia, S.; Chen, L.; Sun, F. Optimal paths for minimizing entransy dissipation during heat transfer processes with generalized radiative heat transfer law. Appl. Math. Model. 2010, 34, 2242-2255.

41. Rozonoer, L.I. A generalized thermodynamic approach to resource exchange and allocation. I. Autom. Rem. contr. 1973, 5, 781-795.

42. Rozonoer L.I. A generalized thermodynamic approach to resource exchange and allocation. II. Autom. Rem. Contr. 1973, 6, 915-927.

43. Rozonoer, L.I. A generalized thermodynamic approach to resource exchange and allocation. III. Autom. Rem. Contr. 1973, 8, 1272-1290.

44. Berry, R.S.; Andresen, B. Thermodynamics constraints in economic analysis. In Self-Organization and Dissipative Structures: Applications in Physical and Social Sciences; Schieve, W.C., Allen, P.M., Eds.; University of Texas Press: Ausin, TX, USA, 1982.

45. Saslow, W.M. An economic analogy to thermodynamics. Am. J. Phys. 1999, 67, 1239-1247.

46. Salamon, P.; Komlos, J.; Andresen, B.; Nulton, J.D. A geometric view of welfare gains with noninstantaneous adjustment. Math. Soc. Sci. 1987, 13, 153-160.

47. Tsirlin, A.M. Optimal control of resource exchange in economic systems. Autom. Rem. Contr. 1995, 56, 401-408.

48. De Vos, A. Endoreversible thermoeconomics. Energ. Convers. Manag. 1995, 36, 1-5.

49. De Vos, A. Endoreversible economics. Energ. Convers. Manag. 1997, 38, 311-317.

50. De Vos, A. Endoreversible thermodynamics versus economics. Energ. Convers. Manag. 1999, 40, 1009-1019. 
51. Martinas, K. About irreversibility in economics. Open Syst. Inform. Dynam. 2000, 7, 349-364.

52. Tsirlin, A.M. Irreversible microeconomics: optimal processes and control. Autom. Rem. Contr. 2001, 62, 820-830.

53. Tsirlin, A.M.; Kazakov, V.; Kolinko, N.A. Irreversibility and limiting possibilities of macrocontrolled systems: I. Thermodynamics. Open Syst. Inform. Dynam. 2001, 8, 315-328.

54. Tsirlin, A.M.; Kazakov, V.; Kolinko, N.A. Irreversibility and limiting possibilities of macrocontrolled systems: II. Microeconomics. Open Syst. Inform. Dynam. 2001, 8, 329-347.

55. Tsirlin, A.M.; Kazakov, V.A. Optimal processes in irreversible thermodynamics and microeconomics. Interdiscipl. Description Complex Syst. 2004, 2, $29-42$.

56. Amelkin, S.A.; Martinas, K.; Tsirlin, A.M. Optimal control for irreversible processes in thermodynamics and microeconomics. Autom. Rem. Contr. 2002, 63, 519-539.

57. Amelkin, S.A. Limiting possibilities of resource exchange process in complex open microeconomic system. Interdiscipl. Description Complex Syst. 2004, 2, 43-52.

58. Tsirlin, A.M.; Kazakov, V. Optimal processes in irreversible microeconomics. Interdiscipl. Description Complex Syst. 2006, 4, 102-123.

59. Tsirlin, A.M. Problems and methods of averaged optimization. Proc. Steklov Inst. Math. 2008, 261, 270-286.

60. Gyftopoulos, E.P. Fundamentals of analysis of processes. Energ. Convers. Manag. 1997, 38, 1525-1533.

61. Sekulic, D. P. A fallacious argument in the finite time thermodynamics concept of endoreversibility. J. Appl. Phys. 1998, 83, 4561-4565.

62. Moran, M.J. In Proceedings of the (ECOS'98) Efficiency, Cost, Optimization, Simulation and Environmental Aspects of Energy Systems and Processes, Nancy, France, 8-10 July 1998; Bejan, A., Feidt, M., Moran, M.J., Tsatsaronis, G., Eds.; Springer: Berlin, Germany, 1998: Volume II, pp. 1147-1150.

63. Moran, M.J. On second-law analysis and the failed promise of finite-time thermodynamics. Energy 1998, 23, 517-519.

64. Gyftopoulos, E.P. Infinite time (reversible) versus finite time (irreversible) thermodynamics: A misconceived distinction. Energy 1999, 24, 1035-1039.

65. Salamon, P. Physics versus engineering of finite-time thermodynamic models and optimizations. In Thermodynamic Optimization of Complex Energy Systems, NATO Advanced Study Institute, Neptun, Romania, 13-24 July 1998; Bejan, A., Mamut, E., Eds.; Kluwer Academic Publishers: The Netherlands, 1999; pp. 421-424.

66. Salamon, P. A Contrast between the physical and the engineering approaches to finite-time thermodynamic models and optimizations. In Recent Advances in Finite Time Thermodynamics; Wu, C., Chen, L., Chen, J., Eds.; Nova Science Publishers: New York, NY, USA, 1999; pp. 541552.

67. Chen, J.; Yan, Z.; Lin, G.; Andresen, B. On the Curzon-Ahlborn efficiency and its connection with the efficiencies of real heat engines. Energ. Convers. Manag. 2001, 42, 173-181.

68. Salamon, P.; Nulton, J.D.; Siragusa, G.; Andresen, T.R.; Limon, A. Principles of control thermodynamics. Energy 2001, 26, 307-319. 
69. Salamon, P.; Hoffmann, K.H.; Schubert, S.; Berry, R.S.; Andresen, B. What conditions make minimum entropy production equivalent to maximum power production? J. Non-Equibri. Thermodyn. 2001, 26, 73-83.

(C) 2011 by the authors; licensee MDPI, Basel, Switzerland. This article is an open access article distributed under the terms and conditions of the Creative Commons Attribution license (http://creativecommons.org/licenses/by/3.0/). 